\title{
Research on the Method of Task-oriented Programming Instruction Take BV Programming Instruction as an Example
}

\author{
${ }^{\mathrm{a}}$ Huansong Yang , ${ }^{\mathrm{b}}$ Shaobin Song, ${ }^{\mathrm{c}}$ Leilei Chen \\ Hangzhou Institute of Service Engineering HangZhou Normal University Hangzhou, Zhejiang, P.R.China
}

\begin{abstract}
Programming instruction is a difficult point at all times, and many students are fearful of learning programming. Good teaching effect has been obtained if teachers can teach students all knowledge points in syllabus through practical software development process, namely, task-oriented programming instruction.
\end{abstract}

Index Terms: Programming; VB; Task; Instruction

(C) 2012 Published by MECS Publisher. Selection and/or peer review under responsibility of the International Conference on E-Business System and Education Technology

\section{Introduction}

Priority in our educational reform is to develop students' practical abilities, which decide if they can be well employed or not. Moreover, employment is also an important indicator of educational quality. At present, most college students have great ambitions but little talents, or they tend to be much more utilitarian. So good teaching effect has been obtained if teachers can teach students all knowledge points in syllabus through practical software development process. This teaching method is also called task-oriented programming instruction. It not only goes with teaching rules, but also arouses students' motivation.

For many years, most of computer programming teachers in colleges put forward that teachers shall fully use the task-driven teaching method in computer programming. However, seldom have they truly implemented in their instructions. The reason is it is hard to organize curriculum contents. Besides, teachers do not show enough determination and persistence. Instead, they are criticized and blamed by counterparts. So they lack courage, and moreover, they have insufficient materials. Such reasons make the task-driven teaching method difficult to put into effect. Here, two cases about VB programming are only for your reference. 


\section{Case Instruction}

\subsection{Case 1:Use VB to Write Programs of Transposition Matrix}

\section{1) Transposition Matrix Algorithms}

Transposition matrix is a new matrix, which has come from an exchanged matrix determinant.

The transfer matrix of $\left|\begin{array}{cccc}1 & 3 & 5 & 7 \\ 2 & 4 & 6 & 8 \\ 3 & 5 & 7 & 9\end{array}\right|$ is $\left|\begin{array}{lll}1 & 2 & 3 \\ 3 & 4 & 5 \\ 5 & 6 & 7 \\ 7 & 8 & 9\end{array}\right|$

If you want to exchange the matrix determinant, you shall exchange their subscripts. Suppose the original matrix is "a" (the left one), and transposition matrix is "b"( the left one), then the second line with the third list in "a" is a23. At the same time, the third list with the second lie in "b" is b32. Thus, the general expression of the transposition matrix: bij $=$ aji

\section{2) Random Matrix}

As to a matrix, it is hard to manually input all elements. Therefore, you can use VB to automatically generate the random matrix of the $\mathrm{m}$ line with the $\mathrm{n}$ list. And you can get $\mathrm{m}$ and $\mathrm{n}$ through 2 text box control:

'Declare Module

Variable of

Dim A() As Integer

'Dynamicarray

Dim m As Integer, n As Integer

'Lines and lists

Private Sub Command1_Click() 'Amatrix of random numbers

Dim i As Integer, j As Integer

Cls

Randomize

$\mathrm{m}=$ Text1.Text

'Lines of the matrix

$\mathrm{n}=$ Text2.Text

'Lists of the matrix

ReDim A(1 To m, 1 To n)

'Redefine numbers

According to $\mathrm{m}$ and $\mathrm{n}$

Print

For $\mathrm{i}=1$ To $\mathrm{m}$

For $\mathrm{j}=1$ To $\mathrm{n}$

$\mathrm{A}(\mathrm{i}, \mathrm{j})=\operatorname{Int}(\mathrm{Rnd} * 99)+1 \quad$ 'The array of elements generated by random numbers between 1-99

Print Format(A(i, j), "@@@@); 'Print original numbers, and fill four digits.

Next 
Print: Print

'Start a new line, and a blank line shall be added in each line

Next

End Sub

The matrix in Fig 1

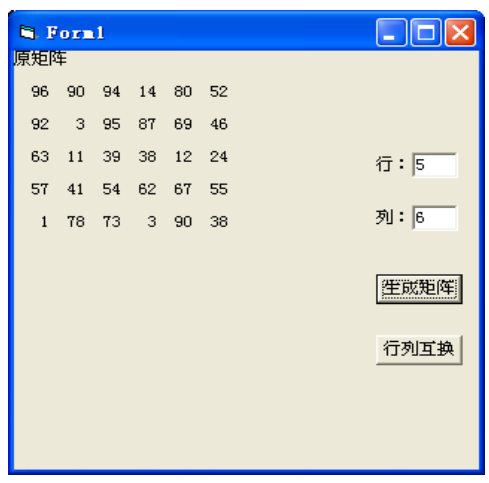

Figure 1. The random matrix of five lines with six lists

\section{3) Transposition of Matrix}

After transposition, lines of matrix are equal to lists of the original matrix. In addition, lists are equal to lines of the original matrix:

Private Sub Command2_Click()

'The button of matrix transportation

Dim i As Integer, j As Integer

Print: Print

For $\mathrm{i}=1$ To $\mathrm{n}$

'Lines are equal to lists of the original matrix

For $\mathrm{j}=1$ To $\mathrm{m}$

'Lists are equal to lines of the original matrix

Print Format(A(j, i), "@@@@); 'Exchange and output lines and lists

Next

Print: Print

Next

End Sub

The matrix after transposition is showed in Fig 2 


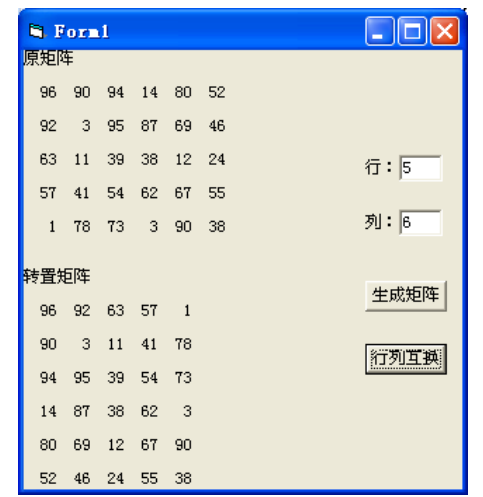

Figure 2. The matrix of six lines and five lists after transposition

\subsection{Case 2: Use VB to write the Yanghui trangle programme}

Yanghui triangle is asked to input yanghui triangle with $\mathrm{n}$ lines in forms like Fig 3

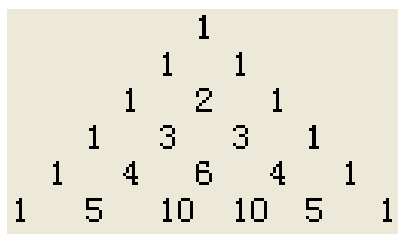

Figure 3. Yanghui Triangle

\section{1) Feature}

Yanghui triangle is founded by Jia Xian (an ancient Chinese mathematician) in the 11th century and it has been stated by Yanghui (an ancient Chinese mathematician in Southern Song Dynasty) in his book. The superficial feature of Yanghui Triangle is that two bevel edges consist of number 1, and other numbers are the sums of two numbers on each top. The internal feature of Yanghui triangle is each coefficient of ( " $n$ " is started from 0$)$ unfolding $(\mathrm{x}+\mathrm{y}) \mathrm{n}$

According to the superficial feature of Yanghui triangle, you can find the rule of numbers: the coefficient in the " $\mathrm{i}$ " line with the " $\mathrm{j}$ " list is the $\mathrm{j}$ unit from " $\mathrm{i}$ ". ( " $\mathrm{i}$ ” and "j" are both started from 0 ).

$C_{m}^{n}=\frac{m !}{n !(m-n) !}$

It means a formula of each coefficient before the binomial is unfolded

\section{2) Programming of Yanghui Triangle}

\section{Interface Design}

Add a tag, a textbox and a button in the upper right corner of the form. Moreover, input layers of Yanghui Triangle in the textbook. The button is used to calculate and output. The interface is shown like Fig4 


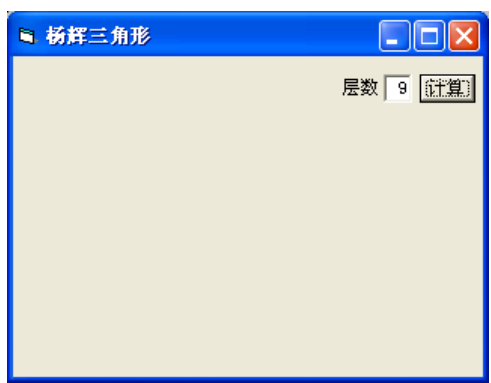

Figure 4. Interface of Yanghui Triangle

(2) Calculation of each number

State a two-dimensional array. Then by using the formula, you can calculate each numerical value and put the array in the corresponding elements.

Private Sub Command1_Click()

Dim n As Integer, i As Integer, j As Integer, a() As Integer 'The dynamic array

$\mathrm{n}=\operatorname{Val}($ Text1.Text) -1 'Get layers and “n”is calculated from 0

ReDim a(n, n) 'Redefine the two-dimensional array

For $\mathrm{i}=0$ To $\mathrm{n} \quad$ 'The subscript of the first dimension

For $\mathrm{j}=0$ To $\mathrm{i} \quad$ ' The subscript of the Second dimension. There are " $\mathrm{i}+1$ ” numbers in " $\mathrm{i}$ ” line, and that is to say, circulate " $\mathrm{i}+1$ ” times

$\mathrm{a}(\mathrm{i}, \mathrm{j})=(\operatorname{Fact}(\mathrm{i}) / \operatorname{Fact}(\mathrm{j}) / \operatorname{Fact}(\mathrm{i}-\mathrm{j})) \quad$ 'Calculate each value through the formula and put it in each array.

Next

Next

display a, $n$

'Display the process and deliver the arrays and layers.

End Sub

In the circulation of ( $i$ ) can you set the circulation of $(j)$. As there are numbers of " $i+1$ "in the line of “ $i$ ", so the circulation of $(\mathrm{j})$ is over as long as “ $\mathrm{j}$ ” is changed from 0 to $\mathrm{i}$.

The formula of calcuating combinational numbers has three multiplies, and it means the factorial function written by one has been used. The code is as follows:

Function Fact(n As Integer) As Long 'Calculation of the factorial function

Dim i As Integer

Fact $=1$

For $\mathrm{i}=1$ To $\mathrm{n}$

Fact $=$ Fact $* \mathrm{i}$

Next

End Function 
The formula is stated like Fact(i) / (Fact(j) * Fact(i - j)), which can be written as Fact(i) / Fact(j) / Fact(i - j).

The last line is display a, n, and it is the process of three triangles. It needs to deliver two parameters, the whole arrays and layers of three triangles.

(2) Printing of Three Triangles

Moreover, format control and output standard Yanghui Triangle. The code is as follows, like fig 5:

Sub display(b() As Integer, $m)$

Dim i As Integer, j As Integer

Cls

For $\mathrm{i}=0$ To $\mathrm{m}$

Print $\operatorname{Tab}(2 *(\mathrm{~m}-\mathrm{i})+1) ; \quad$ 'From bottom upward $\mathrm{Tab}=1,3,5,7,9, \ldots-2 * 0+1,2 * 1+1, \ldots \ldots$

For $\mathrm{j}=0$ To $\mathrm{i}$

Print b(i, j);

If $b(i, j)<10$ Then Print $\operatorname{Spc}(1)$; 'Two spaces after the two-digit number and three spaces fater a digit number

Next

Print

Next

End Sub

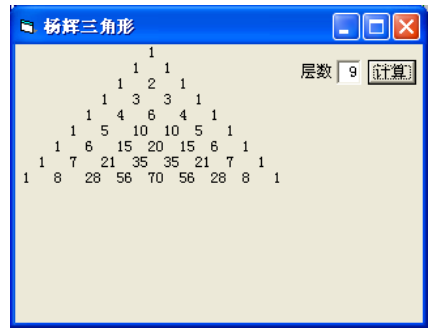

Figure 5. Output of Standard Yanghui Triangle

It is the most difficult for format control is to calculate the starting lists in each printing. According to the rule of Yanghui triangle, the last line is printed from the first list. The penultimate line is printed from the third list. The starting list is added 2 in each line above. Therefore, the rule of the starting list in printing is 1,3 , $5, \ldots . .2 \mathrm{i}+1$ ( " $\mathrm{i}$ " is calculated from 0 ) from bottom upward. On the country, the rule of the starting line in " $\mathrm{i}$ " line is $2(n-i)+1$ from top downward. The "i" in the first line is equal to 0 , so you can print from the list of " $2 n+1$ ". The " $\mathrm{i}$ " in the last line is equal to " $\mathrm{n}$ ", so the result is " 1 ", and it means you can print from the first list.

When you print the numerical data, VB will automatically add a space after the previous number and add a sign digit after a number. It means once the two numbers are positive numbers, there are two spaces between them. If the number in the next line is just pointing to the middle of the two numbers in the last line, then odd spaces is necessary. Thus, after printing a number, we add a space. However, it's only added one space after onedigit number instead of the two-digit number, or it will lead to misplacement rightward. As far as we judge, the space shall be added only when the number is less than 10. (The codes above are correct if they are less 
than 9 layers in Yanghui triangle. If the codes are more than 9 layers, they will have the three-digit number or ever bigger numbers. Therefore, it is necessary to recalculate the format, and it is more complicated.)

\section{Conclusion}

Based upon the tasks, the two cases above both appropriately state new knowledge like static variable, pop-up menus, and relevant parameters. All these are required by the syllabus. Therefore, the teaching instruction is totally different from the traditional programming method. (Students are taught knowledge background first and practical cases afterwards.) Such method is effective and can excide students. It can also help student overcome the fear of leaning programming. Therefore, it is rewarding and advisable for teachers.

\section{References}

[1] Luo chaosheng.Visual Basic programming course (in Chinese). people’s post press,2005

[2] Xu Zhixiang,Liu Liang,Ma Qiang,Wang Xiaotao.Loeal Image Color Components Analysis Method and its Applivcation in online DVD Recorder Measurement[C].Proceedings of 2007 8th International Conference on Eleetronic Measurement \& Instruments.2007,8(2):738-741.

[3] ADLINK Technology Inc.WD-DASK Function Reference Manual(in Chinese). Science Press,Beijing,2007.

[4] Mingyou Xu, Dynamics(in Chinese),Science Press,Beijing,2003

[5] Rafael C.Gonzalez. Digtal Image Processing Second Edition(in Chinese). Publishing House of Electronices Industry,2007

[6] Fan Jiu-lun.Two-dimensional Cross-entropy Linear-type Threshold Segmentation Method for Gray-level Images[J],Acta Electronica Sinica.2009,37(3),pp.450 455 